\title{
Awareness and Perception of National Pharmacovigilance Center among Lebanese Medical Staff
}

Awada Sanaa*, Al-Hajje Amal, Rachid Samar, Mehdi Naïm, Bouzeid Mayssam, Khiami Ghinwa, Bawab Wafaa and Zein Salam

Clinical Pharmacy Department, Faculty of Pharmacy, Lebanese University, Hadath, Beirut, Lebanon

\begin{abstract}
Background: The safety of patients and the safe use of medicines are high requisitions in the modern world, this emerged the practice and science of pharmacovigilance. This study evaluates the opinions and the perceptions about the importance and the need of national Pharmacovigilance center among Lebanese medical staff.

Methods: A cross-sectional study was carried to assess the perception of the healthcare professionals (doctors, pharmacists, nurses, and dentists) working in hospitals, private clinics and community pharmacies regarding the national pharmacovigilance center.

Results: Results showed a $91.27 \%$ response rate. Only $46.2 \%$ of healthcare providers were aware of the pharmacovigilance term. $69.23 \%$ of them gave correct response regarding the definition of pharmacovigilance and $39.90 \%$ of the participants knew the most important purpose of pharmacovigilance. Among the participants, $87.6 \%$ have experienced ADRs in their practice, but only $16.3 \%$ have ever reported ADRs. $12.4 \%$ of the healthcare workers have been trained for reporting adverse reactions, but, $91.6 \%$ healthcare professionals agreed that ADR reporting is necessary and $89 \%$ considered that pharmacovigilance should be taught in details to healthcare professionals.

Conclusion: Pharmacovigilance is being taught to some extent in theory, but the knowledge on the practical approach is lacking. Today, the need for an efficient pharmacovigilance system is necessary more than ever, to ensure the safe use of medicines. An urgent culture of learning about pharmacovigilance taken at the national level should be started earlier.
\end{abstract}

Keywords: Pharmacovigilance; Adverse drug reactions; Awareness; Healthcare providers

\section{Introduction}

The safety of patients and the safe use of medicines are high requisitions in the modern world, this emerged the practice and science of pharmacovigilance [1]. The WHO defines Pharmacovigilance "as the science and activities relating to the detection, assessment, understanding and prevention of adverse effects or any other drug related problems (DRPs)" [2]. Adverse drug reactions (ADRs) reporting is the cornerstone of pharmacovigilance activity. A commonly accepted definition of an ADR is that given by the WHO and recognized in Europe by the International Conference on Harmonization (ICH) as being "a response to a drug which is noxious and unintended and which occurs at doses normally used in man for prophylaxis, diagnosis or therapy of disease or for modification of physiological function" $[3,4]$. The Faculty of Pharmacy at the Lebanese University has created in 2004, a center to survey ADRs in Lebanon, decreed by Council of Ministers President (Decree No/13370/), however, at the present time it is not active anymore. ADRs constitute a significant health problem with consequences for the patient as well as for society [5]. Suspected ADRs have been reported to occur in 2 to $14 \%$ of hospitalized patients and it may have caused or contributed to the fatal outcome [6]. The success of any surveillance system relies on the active participation of its reporters, and is the responsibility of everyone involved in the medication use process [4]. In order to support the activity of the pharmacovigilance programs, the primary objective of the present study was to assess the opinions and the perceptions about the national Pharmacovigilance system among Lebanese medical staff (doctors, pharmacists, dentists, and nurses).

\section{Population and Methods}

A questionnaire based, cross-sectional study was adopted to address the study objective. It was conducted randomly at private clinics, eleven different Lebanese hospitals and community pharmacies in Beirut, North, South, Bekaa and Mount Lebanon. Hospitals and healthcare providers were randomly selected out of a list respectively taken from the ministry of health and medical administration in each selected hospital. The study protocol and questionnaire were approved by the ethic committees of these hospitals. Private clinics and community pharmacies were selected as systemic simple random sample $(1 / 20)$. In fact, an interviewer visited the locations on randomly selected dates and filled the questionnaire. In order to maintain confidentiality about the participants, the entire questionnaire was designed to maintain anonymity. The study was conducted over a period of four months from February to May 2015.

Eligible participants were Lebanese Medical staff including physicians, pharmacists, nurses and dentists. Included participants were subjects who accepted to participate and were graduated healthcare providers.

A questionnaire was drafted based on earlier studies in a way that best suites the Lebanese setting. It was adapted from previous studies on ADR reporting systems in the world to suit the purpose of the

*Corresponding author: Sanaa Awada, Clinical Pharmacy Department, Faculty of Pharmacy, Lebanese University, Hadath, Beirut, Lebanon, Tel: 961-5-463362; Fax: 961-5-463312; E-mail: sanaa3a@hotmail.com

Received: February 18, 2016; Accepted February 24, 2016; Published February 29, 2016

Citation: Sanaa A, Amal AH, Samar R, Naïm M, Mayssam B, et al. (2016) Awareness and Perception of National Pharmacovigilance Center among Lebanese Medical Staff. J Pharmacovigilance 4: 199. doi:10.4172/2329-6887.1000199

Copyright: ( 2016 Sanaa A, et al. This is an open-access article distributed under the terms of the Creative Commons Attribution License, which permits unrestricted use, distribution, and reproduction in any medium, provided the original author and source are credited. 
study and the Lebanese medical staff $[7,8]$. All subjects could answer the questions easily. The questionnaire was administered to healthcare providers randomly selected by the same interviewer. Each question was an open-ended or closed ended question and was asked in the same clear way to all participants.

The questionnaire consisted of forty questions framed under five sections. The first section collects information on socio-demographic data, the second one contains five questions related to knowledge and experience regarding ADR reporting, the third section was designed with sixteen questions, to evaluate the attitude and practice on ADR reporting. The fourth section was made up of six questions that cover various factors, as possible reasons, in the prescriber's perspective that could be discouraging from reporting ADRs. And the last section contains six questions in order to evaluate information with regards to understand the concept of pharmacovigilance and to assess the opinions about the presence and the need of a pharmacovigilance center in Lebanon which is the main objective of the study.

Data collected were analyzed using a commercial computer package SPSS version 21. Results were presented as mean \pm standard deviation (SD) for quantitative variables and numbers with percentages for categorical variables. Bivariate and multivariate analyses were undertaken. The dependent variable for logistic regression was the awareness of pharmacovigilance which is a dichotomized variable. Only variables having a $p<0.2$ in bivariate analysis were included in multivariate analysis. We considered a 95\% confidence interval and a value of $\mathrm{p}<0.05$ to be statistically significant.

\section{Results}

The questionnaire was distributed to 493 healthcare providers out of which 450 agreed to participate, giving a $91.27 \%$ response rate. The study was conducted at eleven different Lebanese hospitals (65.7\%), private clinics (9.2\%) and community pharmacies (25.1\%). Mean age was $35.97(\mathrm{SD}=11)$ and ranged between 20 and 76 years. $56.8 \%$ were males. The majority of the study population was physicians $(51.8 \%)$ and was graduated after 2005 (62.2\%). The participants learned in a variety of countries: 310 (68.9\%) in Lebanon, 71 (15.8\%) in Arab world, $47(10.4 \%)$ in Europe, 14 (3.1\%) in America and 8 (1.8\%) in others (Australia, Iran, Pakistan and turkey). The majority of them studied in the Lebanese University (36.7\%). On average, healthcare providers reported practicing for 9.6 years $(S D=8.9)$ with a minimum of 1 year experience and a maximum of 45 years (Table 1 ).

Fifty six (12.4\%) participants did not observe any ADRs during practicing while 394 participants $(87.6 \%)$ were lucky to observe them. Among healthcare providers who declared their observation of ADRs, the majority of them (55.1\%) had seen less than 10 ADRs in 6 months. Concerning the source of ADR information, the majority received their information from university (31.5\%) and practice $(25.1 \%)$, while others received their knowledge from conferences (13.6\%), pharmaceutical companies (15.3\%), electronic search (11.7\%) and information centers (2.8\%). 17.1\% of the healthcare providers received information about ADRs reporting. Among those, 59 (76.6\%) received written information and only $18(23.4 \%)$ received verbal one from their colleagues (Table 2).

The majority of the participants (91.6\%) believe in the necessity of ADR reporting but only 56 healthcare providers (12.4\%) were trained on reporting an ADR. Those participants were 17 (30.3\%) physicians, 14 (25\%) pharmacists and 25 (44.7\%) nurses. In addition, 107 (23.8\%) declared the availability of ADR reporting forms at their place of work.

\begin{tabular}{|c|c|}
\hline Variables & Mean \pm SD / $\mathbf{n}$ (\%) \\
\hline Age & $35.97 \pm 11$ \\
\hline Gender & \\
Males & $256(56.8)$ \\
Females & $194(43.2)$ \\
\hline Professional status & \\
Physicians & $233(51.8)$ \\
Nurses & $62(13.8)$ \\
Pharmacists & $133(29.6)$ \\
Dentists & $22(4.8)$ \\
\hline Year of graduation & $170(37.8)$ \\
\hline$\leq 2005$ & $280(62.2)$ \\
\hline$>$ 2005 & $310(68.9)$ \\
\hline Country of graduation & $71(15.8)$ \\
Lebanon & $47(10.4)$ \\
Arab world & $14(3.1)$ \\
Europe & $8(1.8)$ \\
\hline America & $165(36.7)$ \\
Others & $145(32.2)$ \\
\hline University & $140(31.1)$ \\
\hline Public university & 9.68 .9 \\
\hline Private university & \\
\hline Foreign universities & \\
\hline Practicing years & \\
\hline
\end{tabular}

Table 1: Sociodemographic characteristics of participants $(n=450)$.

\begin{tabular}{|c|c|}
\hline Variables & $\mathbf{n ~ ( \% )}$ \\
\hline Yes & \\
No & $394(87.6)$ \\
\hline $\begin{array}{c}\text { Have you ever observed or diagnosed an ADR? } \\
\text { If yes, how many ADR on average would be diagnosed } \\
\text { (or observed) in a period of 6 months? } \\
<10\end{array}$ \\
$10-25$ & $217(48.2)$ \\
$25-50$ & $116(25.8)$ \\
$>50$ & $45(10)$ \\
\hline From where did you get your knowledge about ADR? & $16(3.6)$ \\
University & $292(31.5)$ \\
Practice & $232(25.1)$ \\
Conferences & $126(13.6)$ \\
Pharmaceutical companies & $142(15.3)$ \\
Electronic search & $108(11.7)$ \\
Information centers & $26(2.8)$ \\
\hline reporting? \\
Yes \\
No
\end{tabular}

Table 2: Knowledge and experience of medical staff regarding ADR reporting.

Among our 450 healthcare providers, 377 (83.7\%) were unfamiliar with reporting an ADR. While only 25 physicians, 3 dentists, 24 pharmacists and 21 nurses declared reporting ADRs. Almost the half of familiar respondents (53.3\%) sent their reports to the hospital's pharmacy. $42.6 \%$ sent the reports to pharmaceutical companies and 3 physicians (4.1\%) reported ADRs to a pharmacovigilance center while practicing their job outside Lebanon (Table 3).

Most of the healthcare providers (65.7\%) suggested that absence of reporting system is the main factor that discourages them from reporting ADRs, followed by lack of time to report (31.8\%), difficulty to decide whether ADR has occurred (21.8\%), feeling to be exposed to legal liability by reporting ADRs (19.3\%) and $18.2 \%$ do not know how to report. On the other hand only 9 participants (2\%) assumed that only safe drugs are marketed.

The awareness of the term "pharmacovigilance" was evaluated with 
a closed ended question and 242 healthcare providers (53.8\%) were not aware of this term. Among the 208 that claimed knowledge of the term, only 144 (69.23\%) knew its exact meaning which is the detection, assessment, understanding and prevention of ADRs. However, 52 (25.00\%) participants thought that safe, effective and economic use of medicines is the answer. Regarding the most important purpose of the pharmacovigilance, among the 208 participants that claimed knowledge of the term pharmacovigilance, 83 healthcare professionals (39.90\%) knew the most appropriate answer which is to identify safety of drugs (Table 4).

In addition, three hundred eighty nine healthcare providers (86.4\%) were not aware of the existence of a pharmacovigilance center in Lebanon. The rest of our population (61 participants) who knew the presence of such center, were distributed according to their professional status as following: $35(57.4 \%)$ physicians, 23 (37.7\%) pharmacists, $2(3.3 \%)$ dentists and $1(1.6 \%)$ nurse. Out of those 61 healthcare providers, 28 (45.9\%) were graduated from the Lebanese University. In our population, $89 \%$ expressed their positive opinion about the importance of teaching in details the concepts of pharmacovigilance to healthcare professionals and the necessity of having a national pharmacovigilance center in Lebanon.

The association of different variables with awareness of pharmacovigilance was evaluated using bivariate and multivariate analyses. A significant association was seen with different variables in bivariate analyses. It included age $(p=0.045)$, year of graduation $(p=0.002)$, university of graduation $(p=0.013)$, number of practicing years $(\mathrm{p}=0.005)$, continuous education $(\mathrm{p}<0.001)$, being a nurse $(\mathrm{p}=0.001)$, observation or diagnosis of an ADR $(\mathrm{p}=0.09)$, getting knowledge about pharmacovigilance from electronic searches ( $p=0.044)$, thinking that ADR reporting should be necessary $(\mathrm{p}=0.026)$, being familiar with ADR reporting $(\mathrm{p}=0.009)$, updating knowledge about ADR reporting system $(p=0.021)$, considering lifethreatening reactions necessary to report $(p=0.007)$, considering drugs causing hospitalization need to be reported $(p=0.007)$, absence of reporting centers discourages from reporting ADR $(p=0.001)$, exposition to legal liability discourages from reporting $(\mathrm{p}=0.049)$ and knowing the existence of a pharmacovigilance center in Lebanon $(\mathrm{p}<0.001)$.

Results of logistic regression showed that healthcare providers who are continuing their education and those who consider drugs causing hospitalization need to be reported were approximately 2 times more aware of pharmacovigilane (ORa $=1.900,95 \%$ confidence interval [1.244-2.902], $\mathrm{p}=0.003$ and $\mathrm{ORa}=1.485,95 \%$ confidence interval [1.034-2.135], $\mathrm{p}=0.032$ ). In addition, the medical staff who was aware of the existence of a pharmacovigilance center in Lebanon was 27 times more aware of pharmacovigilance $(\mathrm{ORa}=26.905,95 \%$ confidence interval [8.124-49.103], $\mathrm{p}<0.001)$. Also, healthcare providers familiar with ADR reporting and those who consider updating their knowledge about ADR reporting system were approximately 3 times more aware of pharmacovigilance $(\mathrm{ORa}=3.272,95 \%$ confidence interval $[1.765$ 5.063], $\mathrm{p}<0.001$ and $\mathrm{ORa}=2.984,955$ confidence interval [1.1416.803], $\mathrm{p}=0.026$ ). However, being a nurse was approximately 4 times less aware of pharmacovigilance $(\mathrm{ORa}=0.284,95 \%$ confidence interval [0.141-0.572], p < 0.001) (Table 5).

\section{Discussion}

Our study aimed to assess the opinions and the perceptions about the national pharmacovigilance center among Lebanese medical staff (doctors, pharmacists, dentists, and nurses) and to evaluate what

\begin{tabular}{|c|c|}
\hline Variables & $\mathbf{n}(\%)$ \\
\hline $\begin{array}{c}\text { Necessity of ADR reporting } \\
\text { Yes }\end{array}$ & $412(91.6)$ \\
No & $38(8.4)$ \\
Yes & \\
No & $56(12.4)$ \\
\hline Availability of ADR reporting forms at the place of & $394(87.6)$ \\
work & $107(23.8)$ \\
Yes & $343(76.2)$ \\
No & \\
\hline Fes & $73(16.3)$ \\
No & $377(83.7)$ \\
\hline Familiar with ADR reporting & \\
\hline If yes, to whom do you send ADR report? & $39(53.3)$ \\
Hospital's pharmacy & $31(42.6)$ \\
Pharmaceutical companies & $3(4.1)$ \\
\hline Lebarmacovigilance center while practicing outside &
\end{tabular}

Table 3: Attitude and practice on ADR reporting of medical staff.

factors predict a good awareness of the term of pharmacovigilance. Overall, the percentage of healthcare providers classified as aware of the term of pharmacovigilance was 46.2. This percentage is less than that reported for India $[9,10]$ but similar to the results of a study done in Saudi Arabia [11]. These results can elucidate that in the Arab world and especially in Lebanon we are giving neither knowledge, nor importance to pharmacovigilance unlike other Asian countries like India which is one of the most important participants in practicing reporting via pharmacovigilance centers.

The majority of the participants have encountered ADRs. This is similar to results of studies from Nigeria [12] and India [11,13]. But the percentage of the reported ADRs by healthcare professionals was only 16.3. This indicates that there is a huge gap between knowledge and actual reporting; although the national awareness regarding knowledge of ADRs and importance of reporting is high, however the level of reporting ADRs were very low. These findings match the results of a survey done in India by Chopra et al. [14] which revealed that $80 \%$ of healthcare providers had at some point encountered ADR's and only $40 \%$ had ever reported an ADR. This can be due to some factors discouraging reporting ADRs.

These factors responsible for underreporting were also determined in our study. The percentages found in our study among the Lebanese medical staff differ from findings of a survey done in Saudi Arabia that has a constructed well established pharmacovigilance center [15] This difference between the two countries can be due to the working pharmacovigilance center in Saudi Arabia, but both of them states that healthcare providers do not have a clear view of ADR reporting and also shows that the medical staff is not taking his responsibilities seriously towards the danger coming from ADRs. The fact that seriousness of the reactions is the number one encouraging factor and the nonserious reactions are the minor factor, is a normal attitude but unusual reactions according to the Lebanese staff was not an important factor which is opposite to findings from other surveys. In Qatar healthcare pharmacists identified an increased likelihood of reporting a suspected ADR if the reactions were serious for the patient or associated with a new medication [16]. Similar results were obtained by Sanghavi et al. [17], and the participants believed that they should report ADRs if the reaction was serious and unusual in nature. These results raise many questions: why medical staffs do not share the same perception that other healthcare providers have worldwide regarding ADR types that should be reported, and why unusual reactions would not be reported 
Citation: Sanaa A, Amal AH, Samar R, Naïm M, Mayssam B, et al. (2016) Awareness and Perception of National Pharmacovigilance Center among Lebanese Medical Staff. J Pharmacovigilance 4: 199. doi:10.4172/2329-6887.1000199

Page 4 of 5

\begin{tabular}{|c|c|c|c|c|c|}
\hline \multirow[t]{2}{*}{ Pharmacovigilance is the study related to: } & \multicolumn{5}{|c|}{ n (\%) } \\
\hline & Physicians & Pharmacists & Nurses & Dentists & Total \\
\hline 1.Safe,effective and economic use of medicines & $25(21.36)$ & $20(30.30)$ & $6(37.50)$ & $1(11.11)$ & $52(25.00)$ \\
\hline 2.Therapeutic drug monitoring & $6(5.14)$ & $4(6.06)$ & $2(12.50)$ & $0(0)$ & $12(5.76)$ \\
\hline 3.Detection, assessment, understanding and prevention of ADRs & $86(73.50)$ & $42(63.63)$ & $8(50.00)$ & $8(88.89)$ & $144(69.24)$ \\
\hline Total & $117(100)$ & $66(100)$ & $16(100)$ & $9(100)$ & $208(100)$ \\
\hline Purpose of pharmacovigilance & Physicians & Pharmacists & Nurses & Dentists & Total \\
\hline 1.Identify safety of drugs & $48(41.02)$ & $28(42.42)$ & $4(25.00)$ & $3(33.33)$ & $83(39.90)$ \\
\hline 2.Calculate incidence of ADRs & $13(11.11)$ & $7(10.60)$ & $4(25.00)$ & $1(11.11)$ & $25(12.01)$ \\
\hline 3.Identify predisposing factors to ADR & $38(32.47)$ & $22(33.33)$ & $5(31.25)$ & $5(55.55)$ & $70(33.65)$ \\
\hline 4.Identify previously unrecognized ADRs & $18(15.38)$ & $9(13.63)$ & $3(18.75)$ & $0(0.00)$ & $30(14.44)$ \\
\hline Total & $117(100)$ & $66(100)$ & $16(100)$ & $9(100)$ & $208(100)$ \\
\hline
\end{tabular}

Table 4: Influence of professional status on knowledge concerning definition and purpose of Pharmacovigilance.

\begin{tabular}{|c|c|c|}
\hline Variables & ORa & 95\% Cl \\
\hline Continuing education & 1.900 & $1.244-2.902$ \\
\hline Nurse & 0.284 & $0.141-0.572$ \\
\hline Are you familiar with ADR reporting? & 3.272 & $1.765-5.063$ \\
\hline $\begin{array}{c}\text { Would you consider updating your knowledge about ADR reporting } \\
\text { system? }\end{array}$ & 2.984 & $1.141-6.803$ \\
\hline Are drugs causing hospitalization need to be reported? & 1.485 & 0.001 \\
\hline Is there a pharmacovigilance center in Lebanon? & 26.905 & 0.026 \\
\hline
\end{tabular}

Table 5: Multivariate analysis: Binary logistic regression: Aware of the term of pharmacovigilance as dependent variable and predictors as independent variables.

although the unexpected and rare reactions are the most dangerous.

Regarding the ADR reporting center, the percentage of healthcare providers who were aware of the existence of a pharmacovigilance center in Lebanon is similar to findings from studies form Pakistan [18].

Sociodemographic factors such as age, year of graduation and continuous education were associated with a variation of the awareness of the term of pharmacovigilance. Younger healthcare providers were aware of the term of pharmacovigilance. This is similar to findings from a study from Malaysia [19]. However, this inverse relationship was not significant in a study done by Gupta P et al. [20]. Other previous studies have reported either no effect or a direct relationship between age and knowledge $[21,22]$. It can be due to the recent changes in the prospectus handbook like increasing introduction of pharmacovigilance in the undergraduate.

Gender had no effect on knowledge regarding pharmacovigilance, in line with previous Swedish and Malaysia studies [15,21]. However, a study conducted in Nepal reported significantly better questionnaire score in males [22]. Continuous education was found to be a factor that increases awareness of pharmacvigilance. This match results of studies done in India [23] and Germany [24].

Several limitations can be addressed to our study. This study remains a pilot survey with a small sample size that could not extrapolate our results to the national level. Also the influence of the interviewer on healthcare providers' answers can be added as another limitation in such type of studies.

\section{Conclusion}

Our study elucidated several factors that may impact the awareness of pharmacovigilance among Lebanese medical staff. These results suggest a need to improve the practice of pharmacovigilance in the best interest of public safety in this country.
We recommended inclusion of pharmacovigilance in the undergraduate curriculum for healthcare professionals, conducting periodically educational lectures with good emphasis on the concept of pharmacovigilance and the concept of ADRs, sending warning letters or notifications to healthcare professionals regarding serious ADRs associated with drugs, immediately upon obtaining information from drug authorities / Pharmacovigilance centers, facilitating accessibility to ADR reporting forms for example spontaneous reporting of adverse drug reactions through electronic submission and activating the pharmacovigilance center at the Faculty of Pharmacy in the Lebanese University.

\section{References}

1. Bankowski Z, Dunne JF (1993) Drug surveillance: International co-operation past, present and future. Proceedings of the XXVIIth CIOMS Conference, Geneva, Switzerland.

2. Blenkinsopp A, Wilkie P, Wang M, Routledge PA (2007) Patient reporting of suspected adverse drug reactions: a review of published literature and international experience. $\mathrm{Br} \mathrm{J}$ Clin Pharmacol 63: 148-156.

3. World Health Organization (2002) The importance of pharmacovigilance-safety monitoring of medicinal products.

4. Herdeiro MT, Figueiras A, Polónia J, Gestal-Otero JJ (2005) Physician's attitudes and adverse drug reaction reporting : a case-control study in Portugal. Drug Saf 28: 825-833.

5. Beard K (1992) Adverse reactions as a cause of hospital admission in the aged Drugs Aging 2: 356-367.

6. Classen DC, Pestotnik SL, Evans RS, Lloyd JF, Burke JP (1997) Adverse drug events in hospitalized patients. Excess length of stay, extra costs, and attributable mortality. JAMA 277: 301-306

7. Chopra D, Wardhan N, Rehan HS (2011) Knowledge, attitude and practices associated with adverse drug reaction reporting amongst doctors in a teaching hospital. Int J Risk Saf Med 23: 227-232.

8. Jeetu G, Anusha G (2010) Pharmacovigilance: a worldwide master key for drug safety monitoring. J Young Pharm 2: 315-320.

9. Nandha R, Gupta A, Hashmi A (2011) Cutaneous adverse drug reactions in a tertiary care teaching hospital: A North Indian perspective. Int J Appl Basic Med Res 1: 50-53. 
Citation: Sanaa A, Amal AH, Samar R, Naïm M, Mayssam B, et al. (2016) Awareness and Perception of National Pharmacovigilance Center among Lebanese Medical Staff. J Pharmacovigilance 4: 199. doi:10.4172/2329-6887.1000199

10. Pimpalkhute SA, Jaiswal KM, Sontakke SD, Bajait CS, Gaikwad A (2012) Evaluation of awareness about pharmacovigilance and adverse drug reaction monitoring in resident doctors of a tertiary care teaching hospital. Indian $\mathrm{J}$ Med Sci 66: 55-61.

11. Abdel-Latif MM, Abdel-Wahab BA (2015) Knowledge and awareness of adverse drug reactions and pharmacovigilance practices among healthcare professionals in Al-Madinah Al-Munawwarah, Kingdom of Saudi Arabia. Saudi Pharm J 23: 154-161.

12. Bello SO, Umar MT (2011) Knowledge and attitudes of physicians relating to reporting of adverse drug reactions in Sokoto, north-western Nigeria. Ann Afr Med 10: 13-18.

13. Kamtane RA, Wardhani VJ (2012) Knowledge, attitude and perception of physicians towards adverse drug reaction (ADR) reporting: a pharmacoepidemiological study. Asian J Pharm Clin Res 5: 210-214.

14. Chopra D, Wardhan N, Rehan HS (2011) Knowledge, attitude and practices associated with adverse drug reaction reporting amongst doctors in a teaching hospital. Int J Risk Saf Med 23: 227-232.

15. Elkalmi RM, Hassali MA, Ibrahim MI, Widodo RT, Efan QM, et al. (2011) Pharmacy students' knowledge and perceptions about pharmacovigilance in Malaysian public universities. Am J Pharm Educ 75: 96.

16. Wilbur K (2013) Pharmacovigilance in Qatar: a survey of pharmacists. East Mediterr Health J 19: 930-935.

17. Sanghavi DR, Dhande PP, Pandit VA (2013) Perception of pharmacovigilance among doctors in a tertiary care hospital: influence of an interventional lecture. Int J Risk Saf Med 25: 197-204.
18. Iffat W, Shakeel S, Rahim N, Anjum F, Near S, et al. (2014) Pakistani physicians' knowledge and attitude towards reporting adverse drug reactions. African Journal of Pharmacy and Pharmacology 8: 379-385.

19. Salwani BI, Nor Iza AR, Nadiger HA, Wan Putri EWD, Badar UU, et al. (2015) Awareness of pharmacovigilance among future house officers; a Study among first batch of final year medical students of UniSZA, Malaysia. Int J of Pharm Res 7: 96-101.

20. Gupta P, Anvikar AR, Valecha N, Gupta YK (2014) Pharmacovigilance practices for better healthcare delivery: knowledge and attitude study in the national malaria control programme of India. Malar Res Treat 2014: 837427.

21. Ekman E, Bäckström M (2009) Attitudes among hospital physicians to the reporting of adverse drug reactions in Sweden. Eur J Clin Pharmacol 65: 43-46.

22. Palaian S, Ibrahim MI, Mishra P (2011) Health professionals' knowledge, attitude and practices towards pharmacovigilance in Nepal. Pharm Pract (Granada) 9: 228-235.

23. Rajesh R, Vidyasagar S, Varma DM (2011) An educational intervention to assess knowledge attitude practice of pharmacovigilance among health care professionals in an Indian tertiary care teaching hospital. International Journal of PharmTech Research 3: 678-692.

24. Tabali M, Jeschke E, Bockelbrink A, Witt CM, Willich SN, et al. (2009) Educational intervention to improve physician reporting of adverse drug reactions (ADRs) in a primary care setting in complementary and alternative medicine. BMC Public Health 9: 274. 